
\title{
25 Research Square \\ Pentixafor as a theranostic agent in rectal and pancreatic adenocarcinoma: outcomes from a pilot study
}

\section{Elizabeth Ahern}

Royal Brisbane Hospital: Royal Brisbane and Women's Hospital

\section{Paul Thomas}

University of Queensland - Saint Lucia Campus: The University of Queensland

\section{Louise Campbell}

Royal Brisbane Hospital: Royal Brisbane and Women's Hospital

\section{Melissa Latter}

Royal Brisbane and Woman's Hospital Health Service District: Royal Brisbane and Women's Hospital

\section{Melissa Eastgate}

Royal Brisbane Hospital: Royal Brisbane and Women's Hospital

\section{David Wyld}

Royal Brisbane Hospital: Royal Brisbane and Women's Hospital

\section{David A Clark}

Royal Brisbane Hospital: Royal Brisbane and Women's Hospital

\section{Andrew R.L Stevenson}

Royal Brisbane and Women's Hospital

\section{Nicholas O'Rourke}

Royal Brisbane Hospital: Royal Brisbane and Women's Hospital

\section{David Cavallucci}

Royal Brisbane Hospital: Royal Brisbane and Women's Hospital

\section{Richard Bryant}

Royal Brisbane Hospital: Royal Brisbane and Women's Hospital

Matthew Burge ( $\square$ matthew.burge@health.qld.gov.au )

Royal Brisbane Hospital: Royal Brisbane and Women's Hospital https://orcid.org/0000-0003-25627806

\section{Research Article}

Keywords: pentixafor, rectal, pancreatic, adenocarcinoma

Posted Date: April 6th, 2022 
DOI: https://doi.org/10.21203/rs.3.rs-1274952/v2

License: (c) (1) This work is licensed under a Creative Commons Attribution 4.0 International License. Read Full License 


\section{Abstract}

Background: Accurate staging is essential in the management of pancreatic and rectal adenocarcinomas, and where prognosis after management of advanced, unresectable disease remains poor, further treatment options are urgently needed. CXCR4 is the receptor for chemokine CXCL12, with enriched expression in malignant compared with normal tissue, and putative tumorigenic signalling functions. Pentixafor, a peptide targeting CXCR4, is a theranostic agent capable of being labelled with gallium-68 (Ga-68) for positron emission tomography (PET) imaging, and lutetium-177 (Lu-177) for therapy. Using PET scanning, we sought to explore the potential utility of pentixafor as a theranostic agent by examining avidity of Ga-68-pentixafor uptake in a pilot study of patients diagnosed with rectal or pancreatic ductal adenocarcinoma (PDAC).

Methods: We performed a single-arm, prospective, single-institution pilot study. Patients with newly diagnosed pancreatic or rectal adenocarcinoma received Ga-68-pentixafor-PET in addition to the usual clinical and radiologic workup for their disease. Suitability for peptide receptor radionucleotide therapy (PRRT) was judged using the Thera-P trial criteria[1]. Using binomial probability, a target of 12 patients was set for recruitment for each cancer cohort, given that if more than seven patients demonstrated insufficient uptake, there would be $95 \%$ confidence that a sensitivity threshold which would be deemed to warrant further investigation in a larger study (85\%) would not be met.

Results: Pentixafor-PET was administered to 8 rectal cancer patients and 9 PDAC patients and no adverse events were noted. Low-to-moderate levels of pentixafor tracer avidity was demonstrated at all sites of primary disease, most sites of nodal metastatic disease and some distant metastases in each cohort, although less uptake relative to Fluorodeoxyglucose (FDG)-PET/CT was noted suggesting that use of pentixafor is inferior to FDG in staging. No patients satisfied PRRT suitability criteria and further recruitment was terminated.

Conclusion: In this small pilot study, insufficient pentixafor avidity was demonstrated in cases of rectal cancer and PDAC to warrant further exploration as a potential candidate for radionuclide therapy.

Trial registration: Australian New Zealand Clinical Trials Registry. ACTRN12617000596303 (rectal). ACTRN12617000595314 (pancreatic). Registered on 27 th April 2017.

https://www.anzctr.org.au/TrialSearch.aspx.

First patient was enrolled on $2^{\text {nd }}$ May 2017

\section{Introduction:}

The optimal treatment for localized gastrointestinal and hepatobiliary cancers, including rectal and pancreatic cancer, includes surgical resection, where possible. However, for these cancers, surgery may be associated with considerable potential morbidity[2-5]. Furthermore, with the exception of potentially 
curable oligometastatic rectal cancer, there is no standard role for resecting the primary malignancy in the setting of metastatic disease[6, 7].

Survival after diagnosis of pancreatic ductal adenocarcinoma (PDAC) remains very poor, particularly in the metastatic setting, meaning that new treatment approaches are urgently needed. In the case of localised pancreatic ductal adenocarcinoma (PDAC) the chance of relapse post-resection is high, even with contemporary adjuvant or neoadjuvant chemotherapy, implying that a significant proportion of patients with ostensibly localised disease in fact have undiagnosed metastatic disease[8-10]. Detection of this would spare those patients from futile, potentially morbid surgery, and indicate a switch to systemic treatment. Fluorodeoxyglucose positron emission tomography (FDG-PET/CT) has added to conventional workup by improving the detection of metastatic disease: we have shown this changed management in $16 \%$ of cases, previously thought to be suitable for curative resection. [11]. Nonetheless, FDG PET scans are less-well established in staging of pancreas adenocarcinoma compared with their role in staging colorectal adenocarcinomas.

In comparison, relapse rates of rectal adenocarcinoma post-resection are lower, with better long-term survival compared with PDAC, although accurate pre-operative staging in this disease is also essential to plan appropriate perioperative therapy to maximize long-term outcomes. Those patients with rectal cancer with a higher baseline risk of recurrence post-resection, for example with a threatened or involved circumferential resection margin (CRM), locoregional lymph node metastasis or extramural venous invasion, benefit from pre-operative radiotherapy (with pre- and/or post-operative chemotherapy); such benefits are larger than if radiotherapy is given post-operatively, highlighting the need for accurate preoperative staging $[12,13]$. Current standard-of-care pre-treatment imaging in rectal cancer involves a CT of the chest, abdomen and pelvis as well as MRI scanning of the pelvis. In the modern era, more patients relapse with distant disease than local disease, again highlighting the need for accurate baseline imaging to determine stage of disease[7]. The role of baseline FDG-PET/CT in the workup of rectal adenocarcinoma is unclear and does not appear to correlate with endpoints such as overall survival[14]. However, the concept of using PET imaging to differentially assess response to neoadjuvant therapy appears promising[15].

C-X-C chemokine receptor type 4 (CXCR4) is a member of the chemokine receptor family which mediates chemotaxis of cells towards a gradient of chemokines; C-X-C motif chemokine 12 (CXCL12) is the chemokine ligand for CXCR4. CXCR4 has several physiological functions in immunity, embryogenesis, haematopoesis and neoangiogenesis[16]. Furthermore, CXCR4 has been implicated in several pathogenic pathways, most prominently as the co-entry receptor for human immunodeficiency virus-1, but also in autoimmunity and CNS diseases[16]. In oncology, a role for the CXCR4/CXCL12 axis was first uncovered in cancer biology through the observation that CXCR4 protein is overexpressed by multiple human tumour types, initially in breast cancer but including colorectal and pancreatic invasive and in-situ malignancies, and evaluable through immunohistochemistry[17-22]. However, this axis has been further implicated in tumorigenesis, metastasis and tumour immunity, including in pancreatic and colorectal cancers[23]. For example, human pancreatic cancer cells in vitro demonstrate increased survival and proliferation when 
expressing CXCR4, and CXCR4-dependent signalling induces invasion and epithelial-mesenchymal transformation[24, 25]. In preclinical cancer models including pancreatic and colon cancer, interaction between CXCR4 expressed by tumour cells and CXCL12 expressed in the potential metastatic niche in target organs such as bone marrow, lungs, liver and lymph nodes was implicated in metastatic development, and which could be antagonised pharmacologically in some settings[17, 26-28]. As well as these direct anti-cancer activities observed by blocking CXCR4, a synergistic effect with immunotherapy has been demonstrated with CXCL12 antagonism in a mouse model of pancreatic cancer, highlighting a suppressive role of CXCR4/CXCL12 in the tumour-immune microenvironment[29]. CXCR4 expression at a protein level has been investigated in some human cancers for an association with outcome. In a study of resection of hepatic metastases arising from colorectal cancer, and a second independent study of colorectal primary and liver metastases, CXCR4 expression was associated with diminished survival[19, 20]. Furthermore, CXCR4 expression may be associated with poor survival in resected pancreatic cancer[22]. Taken together, the enriched expression of CXCR4 at a protein level in cancer suggests its potential use as a diagnostic biomarker for quantitative imaging, while the functional understandings of CXCR4-dependent signalling further suggests a potential therapeutic role of CXCR4 blockade in anticancer therapy.

Peptide receptor radionucleotide therapy (PRRT), where imaging biomaterials are also used as targeted drug carriers, is situated at this nexus between diagnostic imaging and therapeutics ('theranostics'). Peptide receptor radionucleotide therapy (PRRT) has recently demonstrated advantages compared with standard-care chemotherapy in the setting of metastatic prostate cancer, introducing PRRT as a potentially feasible and efficacious option in solid organ malignancies[1]. CXCR4 has been explored as a target for PET scanning, with the use of pentixafor as the high-affinity nuclear probe in human cancers[30]. Furthermore, whole-body distribution and radiation dosimetry in humans has also been performed[31]. However, data relating to the performance of this tracer in rectal and pancreatic adenocarcinoma is lacking. Given the potential utility of pentixafor as a staging modality and as a PRRT biomaterial, we aimed to describe this tracer in patients with rectal and pancreatic adenocarcinoma of any stage: to evaluate uptake in primary and metastatic sites, to compare with FDG uptake and therefore infer suitability for using pentixafor in a theranostic approach.

\section{Methods:}

\section{Patient cohorts}

Patients were eligible for inclusion in the pilot study if they had either of the following newly-diagnosed biopsy-proven malignancies: rectal adenocarcinoma with the primary tumour located within $15 \mathrm{~cm}$ of the anal verge and below the peritoneal reflection (rectal cancer cohort), or pancreatic ductal adenocarcinoma having received any prior treatment. Malignancies could be of any stage. Exclusion criteria included histopathology other than adenocarcinoma (e.g., neuroendocrine tumour), locally recurrent tumours, ampullary or biliary tumours, unable to undergo a PET scan, pregnancy or breastfeeding. 


\section{Study design}

This single-arm, prospective, single-institution pilot study was conducted for each tumour type (rectal cancer and PDAC cohorts) at the Herston Imaging Research Facility (HIRF) of the Royal Brisbane and Women's Hospital (RBWH), Queensland, Australia. The pilot study opened for recruitment in March 2017 and closed February 2020. The aim of the study was to gain preliminary information on the uptake characteristics and hence theranostic utility of pentixafor PET/CT in each malignancy cohort, by measuring the SUV of the tracer in primary tumour and metastases. In addition, the uptake seen on pentixafor PET/CT was compared with standard imaging studies (including diagnostic CT, ${ }^{18} \mathrm{~F}-\mathrm{FDG}$ PET/CT in the PDAC cohort, and pelvic MRI in the rectal cancer cohort) and monitored adverse events of participants receiving pentixafor PET/CT. The findings of each pentixafor PET/CT were available to the clinicians.

\section{Study procedures}

${ }^{68} \mathrm{Ga}-\mathrm{CPCR} 4-2 \mathrm{PET} / \mathrm{CT}$ (hereafter called pentixafor PET/CT) was performed on Siemens Biograph mCT Flow. The imaging protocol called for images to be acquired 1 hour post intravenous injection of ${ }^{68} \mathrm{Ga}$ CPCR4-2 using standard activity of $150 \mathrm{MBq}$, with scan range from vertex to mid-thigh acquired using 3 minutes per bed position. A low dose CT (LDCT) scan was acquired for the purpose of attenuation correction of PET images and anatomical localization. In the pancreatic cohort, an ${ }^{18} \mathrm{~F}-\mathrm{FDG}$ PET/CT (FDG $\mathrm{PET} / \mathrm{CT}$ ) was additionally performed on Siemens Biograph mCT Flow. The imaging protocol called for FDG images to be acquired 1-hour post injection of ${ }^{18} \mathrm{~F}-\mathrm{FDG}$ using the standard dose $4.5 \mathrm{MBq} / \mathrm{kg}$ (to a maximum $370 \mathrm{MBq}$ ) with scan range from vertex to mid-thigh, with a LDCT scan additionally performed. For adverse event (AE) recording, the Common Terminology Criteria for Adverse Events (CTCAE) version 4.0 was used. The relationship between $\mathrm{AE}$ to the investigational procedure (pentixafor PET/CT scan) was assessed by the treating investigator. Standard definitions were used to define serious AE (SAE). AEs and SAEs were recorded from the time of consent to 30 days after PET scan or the commencement of anti-cancer treatment, whichever came first. AEs were followed until stabilization or resolution.

\section{Statistical considerations}

For this pilot study, a threshold for sensitivity of $85 \%$ was set as sufficient to warrant further investigation in a larger prospective study. For each tumour cohort, a target of 12 patients was set for recruitment, given that if more than seven patients demonstrated insufficient uptake, there would be $95 \%$ confidence that this sensitivity threshold would not be met. Determination of sufficient or insufficient avidity was adapted from the Thera-P trial criteria for a theranostic treatment in metastatic prostate cancer. In that trial, an individual case was determined to be suitable for a theranostic approach if (1) at least one tumour deposit had maximum standardised uptake value (SUVmax) of 20 or more; (2) all other tumour deposits of at least $10 \mathrm{~mm}$ size had SUVmax of at least 10; and (3) experimental radiotracer avidity (measured via SUVmax) was at least equivalent to FDG avidity in all malignant lesions (where FDG-PET was performed). Column analysis for paired group measures was performed via the Wilcoxon matched- 
pairs signed rank test using GraphPad Prism version 9.0.2 San Diego, California with significance considered $p<0.05$.

\section{Ethical considerations}

Radiation dose assessment was based on human dosimetry studies and verified by two medical physicists. The combined total effective radiation dose per participant in the pancreatic cohort undergoing both the pentixafor and FDG examinations was $22 \mathrm{mSv}$, based on a $70 \mathrm{~kg}$ participant, and in the rectal cohort was $9.2 \mathrm{mSv}$. The pilot study was approved by the RBWH Human Research Ethics Committee (HREC/16/QRBW/549 for pancreatic cohort, HREC/16/QRBW/616 for rectal cohort) and was performed in accordance with the National Health and Medical Research Council (Australia) Statement of Ethical Conduct in Research Involving Humans (2007) and the Declaration of Helsinki (2008). This work is registered with the Australian New Zealand Clinical Trials Registry. Registration numbers are: ACTRN12617000596303 (rectal) and ACTRN12617000595314 (pancreatic).

\section{Results:}

\section{Patient characteristics}

8 patients were recruited in the rectal cancer cohort and 9 in the PDAC cohort. The patient characteristics at baseline are shown in Table 1. The median age was 64 years. Most patients in each group had ECOG performance status 1. Staging was performed for each cancer according to American Joint Committee on Cancer (AJCC) 8th edition. In the pancreatic cohort, most patients (7/9) had metastatic disease at presentation whereas two had locally advanced inoperable disease.. In the rectal cohort, six patients had stage 2-3 disease at enrolment, with the other two having stage 4 disease (one with biopsy-proven metastases to inguinal lymph nodes and the other with multiple liver metastases). Prior to having the pentixafor PET/CT, two rectal cancer patients had already commenced chemotherapy (one of which was capecitabine concurrent with radiotherapy), whereas three pancreatic cancer patients had received prior chemotherapy. No patients had undergone surgery for their cancer by the time of pentixafor PET/CT scan. All patients successfully underwent pentixafor PET/CT scan and no trial related adverse events were recorded. All nine pancreatic cancer patients successfully underwent FDG PET/CT and all FDG $\mathrm{PET} / \mathrm{CT}$ scans were performed contemporaneously with pentixafor PET/CT scans

\section{Tracer uptake at primary sites}

Illustrative pentixafor PET scans for rectal cancer and PDAC are shown in Fig. 1A-B and C. In the rectal cancer cohort, all (8/8) patients demonstrated low-to-moderate pentixafor avidity at the primary tumour site, but the SUVmax was less than 10 in all cases (Figs. 1A and 2A). Where performed as part of routine clinical care, all (4/4) cases had higher FDG avidity at the primary than pentixafor. Similar findings were seen in the PDAC cohort, where all patients $(9 / 9)$ had a low level of pentixafor avidity at the primary site (SUVmax $<10$ ), and higher avidity was seen using the FDG tracer in all cases. (Figs. 1B and 2B). In the PDAC cohort, the FDG avidity was significantly higher than the pentixafor avidity ( $p=0.0078)$ (Fig. 2$)$. 


\section{Tracer uptake at locoregional nodal sites}

In the rectal cancer cohort, uptake was less with pentixafor than FDG in all cases where FDG was performed (4/4) (Fig. 3A). Of the other four cases where pentixafor-PET was performed alone, two were staged with nodal disease upon review of clinical and MRI findings; of these, only one demonstrated any abnormal pentixafor uptake, and this was of low avidity (SUVmax <5)(Fig. 3A). In the pancreatic cohort, modest avidity was seen in all cases in locoregional nodes using pentixafor tracer and FDG tracer. In four cases, the pentixafor avidity was slightly higher compared with FDG but the reverse was seen in five cases (Fig. 3B and Fig. 1C).

\section{Tracer uptake at distant metastatic sites}

Avidity of the 2-3 most avid metastases for each case comparing pentixafor and FDG tracers for both cohorts is shown in Fig. 4. In a rectal cancer case, metastatic inguinal LN uptake was evident using pentixafor, although with considerably less avidity than seen using FDG (SUVmax 6.0 vs 24.6). This and another rectal cancer case each had two liver metastases detected with FDG tracer, but these had either low or no avidity using pentixafor. Similarly, in the six PDAC cases where metastatic disease was found on FDG scanning, pentixafor avidity was either lower or absent in all. The total number of metastases detected was higher or equivalent in every patient using FDG compared with pentixafor (Fig. 4), and pentixafor did not detect any confirmed occult metastases which had been missed using conventional modalities. In one case of pancreatic adenocarcinoma, sub-centimetre lung metastases were seen on CT scan but demonstrated no uptake on either PET modality.

\section{Clinical utility of pentixafor tracer as potential theranostic}

No cases in either cohort (PDAC, $n=9$; rectal cancer, $n=8$ ) satisfied the Thera-P theranostic criteria using pentixafor tracer.

\section{Discussion:}

In this pilot study, low-to-moderate levels of pentixafor tracer avidity was demonstrated at all sites of primary disease and most sites of nodal metastatic disease in patients with adenocarcinoma of the rectum or pancreas, albeit at generally lower levels than that seen with FDG. Pentixafor as a diagnostic tracer did not identify occult areas of disease in any patient and detected fewer sites of metastatic disease than FDG tracer. In all participants, Thera-P criteria for theranostic tracer usage was not satisfied by pentixafor. Therefore, with $>95 \%$ confidence that the prespecified threshold for sensitivity would not be met per the statistical plan, the pilot study was terminated.

Pentixafor has also been investigated in the setting of oesophageal adenocarcinoma in a mouse model of ex vivo autoradiography and pilot studies in humans with oesophageal adenocarcinoma or squamous cell carcinoma. [32, 33]. In these studies, correlative immunohistochemical (IHC) studies demonstrated that contributors to CXCR4 expression in tumours included immune cells, stromal elements and tumour cells. Although there was no correlation seen in a small subgroup between tumoral IHC expression of 
CXCR4 and pentixafor avidity via PET, the infiltrating immune cells were the largest contributor of CXCR4 expression in both a mouse EAC model and a larger collection of human retrospective EAC primaries [32, 33]. Consistent with this, pentixafor PET scans in the diagnosis of lymphoid malignancies have shown promise, and in some cases have been more diagnostically sensitive than FDG [34, 35].

As defined by the Thera-P criteria, in our small cohort we found insufficient uptake to support a theranostic role for pentixafor. However, the strong biologic rationale of targeting CXCR4 warrants further research. When assessing pentixafor usage in the setting of rectal and pancreatic malignancies, future studies should include histologic assessment of the cells contributing to CXCR4 expression in these malignancies, in particular the immune cells. Both pancreatic and rectal adenocarcinomas are considered immunologically "cold" tumours with non-inflammatory tumour microenvironments [36]. Therefore, a potential cause of generally low pentixafor avidity in these tumours may be a paucity of infiltrating immune cells at baseline. If so, this may guide the optimal usage of this tracer in these diseases, either to functionally assess immune infiltration following therapy (such as immunotherapy or radiotherapy), or as a theranostic agent. Microsatellite unstable tumours are characterised by high levels of immune cell infiltration and might represent a small subset of patients with more promising pentixafor uptake. All our patients had microsatellite stable cancers. In addition, administering concurrent therapies which increase tumour-lymphocyte infiltration, such as checkpoint inhibition, might be worth exploring. If CXCR4 expression defined by pentixafor PET largely reflects immune cell infiltration into the tumour, this modality might also be used prognostically as increased lymphocytic infiltration is a positive prognostic marker in both pancreatic and rectal cancer $[37,38]$. Of course, our results might reflect that pentixafor is simply not a useful tracer to identify CXCR 4 expression in tumours. That being the case, future research into CXCR4 would need to be undertaken with molecules possessing improved binding affinity to CXCR4.

Our study has other limitations. The number of patients enrolled was small. We had originally planned to enrol 12 patients to each cohort, but recruitment was slow, in part due to many of our patients residing some distance from our centre and not willing to be inconvenienced by an additional PET scan. Some patients had received treatment prior to undertaking the PET scan. It is not known if this impacted tracer binding to CXCR4, but limited uptake was seen regardless of prior therapy. In addition, small patient numbers and limited tracer uptake impacted our ability to undertake any meaningful translational research. We do not have data on the immunohistochemical staining characteristics of CXCR4 hence any relationship this may have with PET scan uptake. Again, these should be incorporated into any larger study.

\section{Conclusion:}

This study found limited pentixafor uptake in the small cohorts of rectal and pancreatic adenocarcinomas and did not meet pre-defined criteria for theranostic utility.

\section{Abbreviations:}


RBWH: Royal Brisbane and Womens Hospital

PDAC: pancreatic ductal adenocarcinoma

PET: Positron Emission Tomography

FDG: fluorodeoxyglucose

CRM: circumferential resection margin

CXCR4: C-X-C chemokine receptor type 4

CXCL12: C-X-C motif chemokine 12

PRRT: Peptide Receptor Radionuclide Therapy

HIRF: Herston Imaging Research Facility

LDCT: Low dose computed tomography

SUV: Standard Uptake Value

IHC: Immunohistochemistry

\section{Declarations:}

\section{Funding}

This work was supported by Metro North (Queensland Health) Hospital and Health Services. A grant was awarded from the Herston Imaging Research Facility (HIRF) seed research funding round.

\section{Conflicts of interest}

The authors have no relevant financial or non-financial interests to declare.

\section{Data Availability}

The imaging data acquired during for this work are stored at the Herston Imaging research facility and the Department of Nuclear Medicine at the Royal Brisbane and Women's Hospital. These imaging data are available from Dr Paul Thomas upon reasonable request.

\section{Ethics Approval}

This study was approved by the RBWH Human Research Ethics Committee (HREC/16/QRBW/549 for pancreatic cohort, HREC/16/QRBW/616 for rectal cohort) and was performed in accordance with the 
National Health and Medical Research Council (Australia) Statement of Ethical Conduct in Research Involving Humans (2007) and the Declaration of Helsinki (2008).

\section{Consent to participate}

Informed consent was obtained from all individual participants included in this study.

\section{Consent to Publish}

The authors affirm that human research participants provided informed consent for the publication of images in Figure 1 A, B and C.

\section{Authors contributions}

All authors, EA, PT, LC, ML, ME, DW, DAC, ARLS, NO, DC, RB, MB read, substantively contributed to, and approved the final manuscript. MB ant PT devised the study design and wrote the protocol. EA, ME, DW, DAC, ARLS, NO, DC, RB, MB contributed to patient recruitment. ML and PT oversaw the acquisition of the CXCR4 PET scans. EA wrote the first draft of the paper and did the first data analysis. PT interpreted and reported on the PET scan findings.

\section{Acknowledgements}

These studies were both funded by Royal Brisbane and Women's Hospital (RBWH) Foundation grants funded by Metro North and RBWH Foundation.

This work would also not have been possible without the support from staff at the Medical Oncology research department and staff at the Herston Research Imaging Facility.

\section{References:}

1. Hofman MS, et al., [< sup > 177</sup > Lu]Lu-PSMA-617 versus cabazitaxel in patients with metastatic castration-resistant prostate cancer (TheraP): a randomised, open-label, phase 2 trial. The Lancet, 2021. 397(10276): p. 797-804.

2. $\mathrm{Ng} \mathrm{JL}$, et al. Totally-laparoscopic versus laparoscopic-assisted low anterior resection for rectal cancer: are outcomes different? ANZ J Surg. 2018;88(12):E818-23.

3. Vennix S, et al., Laparoscopic versus open total mesorectal excision for rectal cancer. Cochrane Database Syst Rev, 2014(4): p. Cd005200.

4. Saraee A, et al. Whipple procedure: a review of a 7-year clinical experience in a referral center for hepatobiliary and pancreas diseases. World J Surg Oncol. 2015;13:98-8.

5. Gagner M, Palermo M. Laparoscopic Whipple procedure: review of the literature. J Hepatobiliary Pancreat Surg. 2009;16(6):726-30. 
6. Maggino L, Vollmer CM Jr. Recent Advances in Pancreatic Cancer Surgery. Curr Treat Options Gastroenterol. 2017;15(4):520-37.

7. Smith JJ, Garcia-Aguilar J. Advances and challenges in treatment of locally advanced rectal cancer. J Clin Oncol. 2015;33(16):1797-808.

8. Conroy T, et al. FOLFIRINOX or Gemcitabine as Adjuvant Therapy for Pancreatic Cancer. N Engl J Med. 2018;379(25):2395-406.

9. Neoptolemos JP, et al. Comparison of adjuvant gemcitabine and capecitabine with gemcitabine monotherapy in patients with resected pancreatic cancer (ESPAC-4): a multicentre, open-label, randomised, phase 3 trial. Lancet. 2017;389(10073):1011-24.

10. Muniz Kovtun J, et al. Neoadjuvant therapy outcomes in nonmetastatic pancreatic cancer. J Clin Oncol. 2018;36(4_suppl):501-1.

11. Burge $M$, et al. A prospective, study of the impact of Fluorodeoxyglucose (FDG) positron Emission Tomograpthy with concurrent non-contrast CT scanning (PET/CT) on the management of operable Pancreatic, and Peri-ampullary cancers. HPB. 2015 Jul;17(7):624-31.

12. Roh MS, et al. Preoperative multimodality therapy improves disease-free survival in patients with carcinoma of the rectum: NSABP R-03. J Clin Oncol. 2009;27(31):5124-30.

13. Kapiteijn E, et al. Preoperative radiotherapy combined with total mesorectal excision for resectable rectal cancer. N Engl J Med. 2001;345(9):638-46.

14. Deantonio L, et al. Does baseline [18F] FDG-PET/CT correlate with tumor staging, response after neoadjuvant chemoradiotherapy, and prognosis in patients with rectal cancer? Radiat Oncol. 2018;13(1):211.

15. Fernando $S$, et al. Prognostic utility of serial (18)F-FDG-PET/CT in patients with locally advanced rectal cancer who underwent tri-modality treatment. $\mathrm{Br} J$ Radiol. 2020;93(1105):20190455.

16. Domanska UM, et al. A review on CXCR4/CXCL12 axis in oncology: No place to hide. Eur J Cancer. 2013;49(1):219-30.

17. Müller A, et al. Involvement of chemokine receptors in breast cancer metastasis. Nature. 2001;410(6824):50-6.

18. Smith MCP, et al. CXCR4 Regulates Growth of Both Primary and Metastatic Breast Cancer. Cancer Res. 2004;64(23):8604.

19. Yopp AC, et al. CXCR4 Expression Predicts Patient Outcome and Recurrence Patterns after Hepatic Resection for Colorectal Liver Metastases. Ann Surg Oncol. 2012;19(3):339-46.

20. Kim J, et al. Chemokine Receptor CXCR4 Expression in Colorectal Cancer Patients Increases the Risk for Recurrence and for Poor Survival. J Clin Oncol. 2005;23(12):2744-53.

21. Thomas RM, et al. The chemokine receptor CXCR4 is expressed in pancreatic intraepithelial neoplasia. Gut. 2008;57(11):1555-60.

22. Maréchal R, et al. High expression of CXCR4 may predict poor survival in resected pancreatic adenocarcinoma. Br J Cancer. 2009;100(9):1444-51. 
23. Zlotnik A, Burkhardt AM, Homey B. Homeostatic chemokine receptors and organ-specific metastasis. Nat Rev Immunol. 2011;11(9):597-606.

24. Marchesi F, et al. Increased Survival, Proliferation, and Migration in Metastatic Human Pancreatic Tumor Cells Expressing Functional CXCR4. Cancer Res. 2004;64(22):8420.

25. Li X, et al. SDF-1/CXCR4 signaling induces pancreatic cancer cell invasion and epithelialmesenchymal transition in vitro through non-canonical activation of Hedgehog pathway. Cancer Lett. 2012;322(2):169-76.

26. Wang Z, et al. Blockade of SDF-1/CXCR4 signalling inhibits pancreatic cancer progression in vitro via inactivation of canonical Wnt pathway. Br J Cancer. 2008;99(10):1695-703.

27. Huang EH, et al. A CXCR4 antagonist CTCE-9908 inhibits primary tumor growth and metastasis of breast cancer. J Surg Res. 2009;155(2):231-6.

28. Zeelenberg IS, Ruuls-Van L, Stalle, Roos E. The chemokine receptor CXCR4 is required for outgrowth of colon carcinoma micrometastases. Cancer Res. 2003;63(13):3833-9.

29. Feig $C$, et al. Targeting CXCL12 from FAP-expressing carcinoma-associated fibroblasts synergizes with anti-PD-L1 immunotherapy in pancreatic cancer. Proc Natl Acad Sci U S A. 2013;110(50):20212-7.

30. Vag T, et al. First Experience with Chemokine Receptor CXCR4-Targeted PET Imaging of Patients with Solid Cancers. J Nucl Med. 2016;57(5):741-6.

31. Herrmann K, et al. Biodistribution and radiation dosimetry for the chemokine receptor CXCR4targeting probe 68Ga-pentixafor. J Nucl Med. 2015;56(3):410-6.

32. Fang $\mathrm{H}-\mathrm{Y}$, et al., <em>CXCR4</em> Is a Potential Target for Diagnostic PET/CT Imaging in Barrett's Dysplasia and Esophageal Adenocarcinoma. Clinical Cancer Research, 2018. 24(5): p. 1048-1061.

33. Linde $P$, et al. Pentixafor PET/CT for imaging of chemokine receptor 4 expression in esophageal cancer - a first clinical approach. Cancer Imaging. 2021;21(1):22.

34. Mayerhoefer ME, et al. CXCR4 PET imaging of mantle cell lymphoma using [(68)Ga]Pentixafor: comparison with [(18)F]FDG-PET. Theranostics. 2021;11(2):567-78.

35. Philipp-Abbrederis K, et al. [68Ga]Pentixafor: A Novel PET Tracer for Imaging CXCR4 Status in Patients with Multiple Myeloma. Blood. 2014;124(21):2014-4.

36. Maleki Vareki S. High and low mutational burden tumors versus immunologically hot and cold tumors and response to immune checkpoint inhibitors. J Immunother Cancer. 2018;6(1):157.

37. Orhan A, et al. The prognostic value of tumour-infiltrating lymphocytes in pancreatic cancer: a systematic review and meta-analysis. Eur J Cancer. 2020;132:71-84.

38. Idos GE, et al. The Prognostic Implications of Tumor Infiltrating Lymphocytes in Colorectal Cancer: A Systematic Review and Meta-Analysis. Sci Rep. 2020;10(1):3360.

\section{Table:}

Table 1 is available in the Supplementary Files section. 


\section{Figures}

A) Rectal cancer
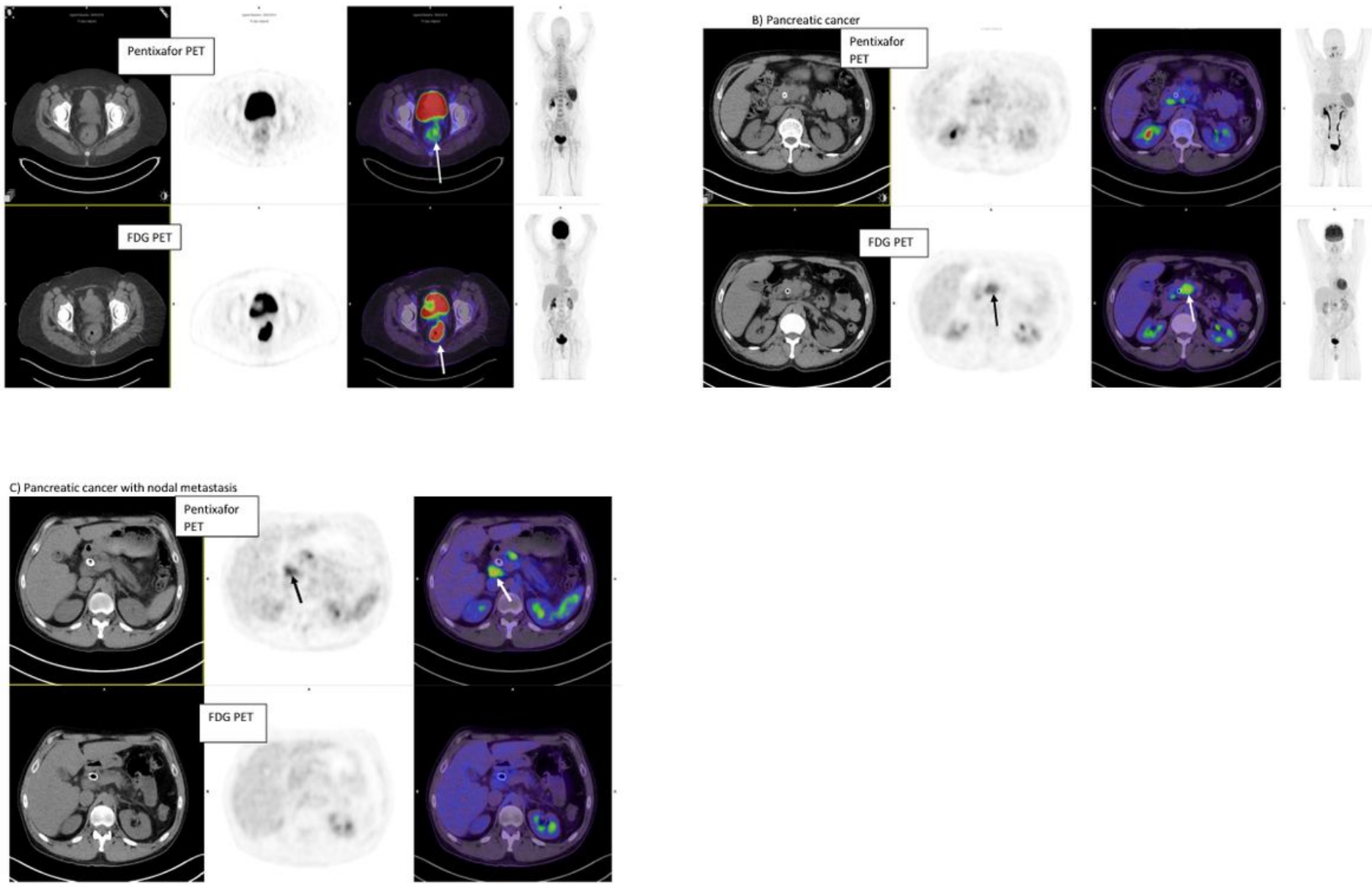

Figure 1

Illustrative PET images. Pentixafor PET top row, FDG PET bottom row (A) a study participant with rectal cancer. Primary tumour arrowed. (B) A study participant with PDAC. Primary tumour uptake. (C) Pentixafor uptake in a nodal metastasis from a participant with PDAC. This is the same patient as in B. 
Figure 2.
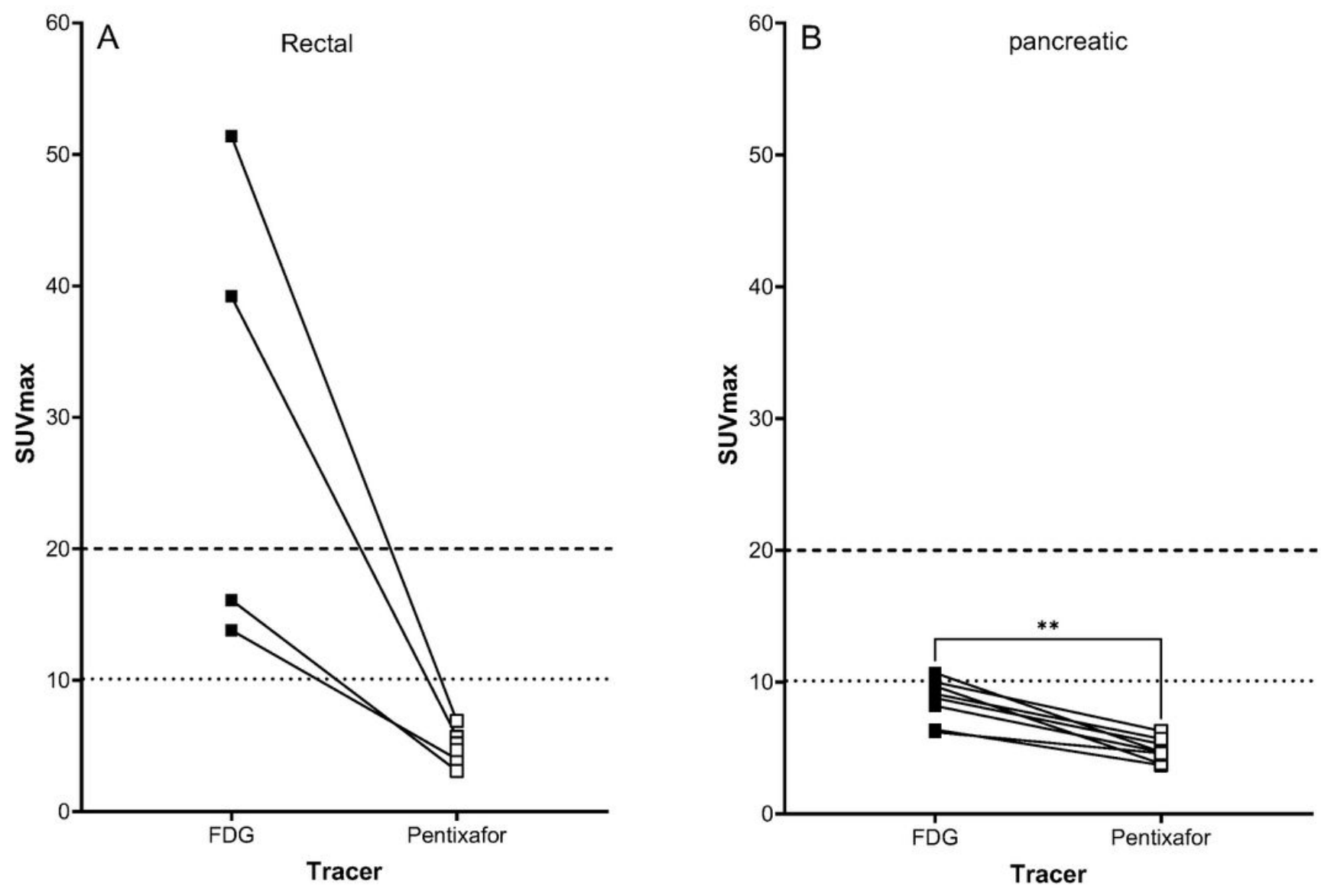

\section{Figure 2}

Maximum standardised uptake value (SUVmax) comparing FDG (where done) and pentixafor tracer in primary tumor site for the following cohorts: (A) rectal cancer and (B) PDAC. Dotted and dashed horizontal lines indicate SUVmax of 10 and 20, respectively. Differences between SUVmax where significant are shown (** $P<0.01)$. 
Figure 3.
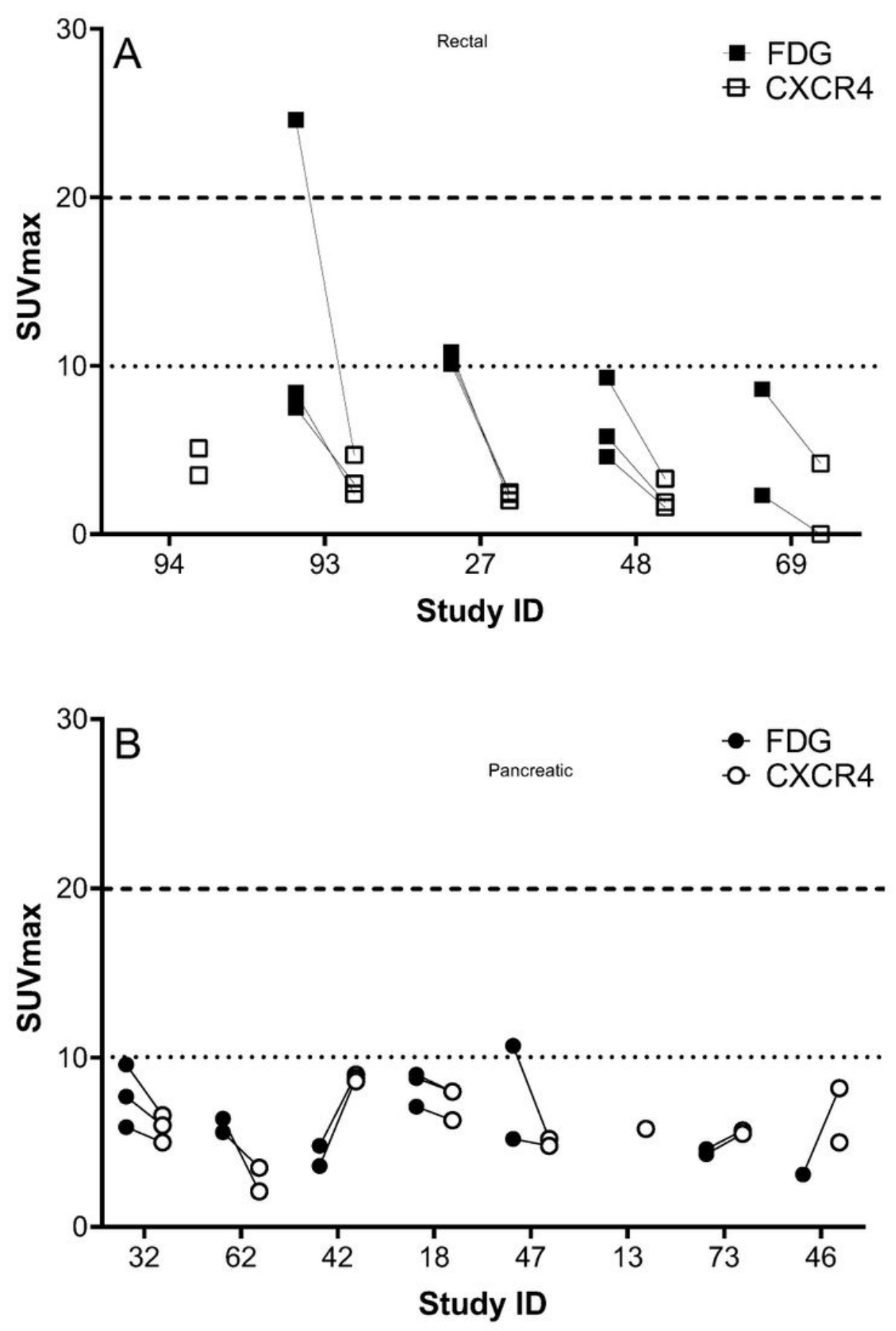

Figure 3

Maximum standardised uptake value (SUVmax) comparing FDG (where done) (closed symbol) and pentixafor tracer (open symbol) in locoregional lymph node metastatic sites for individual study participants (as indicated by the study identification number: Study ID) from (A) rectal cancer and (B) PDAC cohorts where these were detected. Dotted and dashed horizontal lines indicate SUVmax of 10 and 20 , respectively. 
Figure 4.

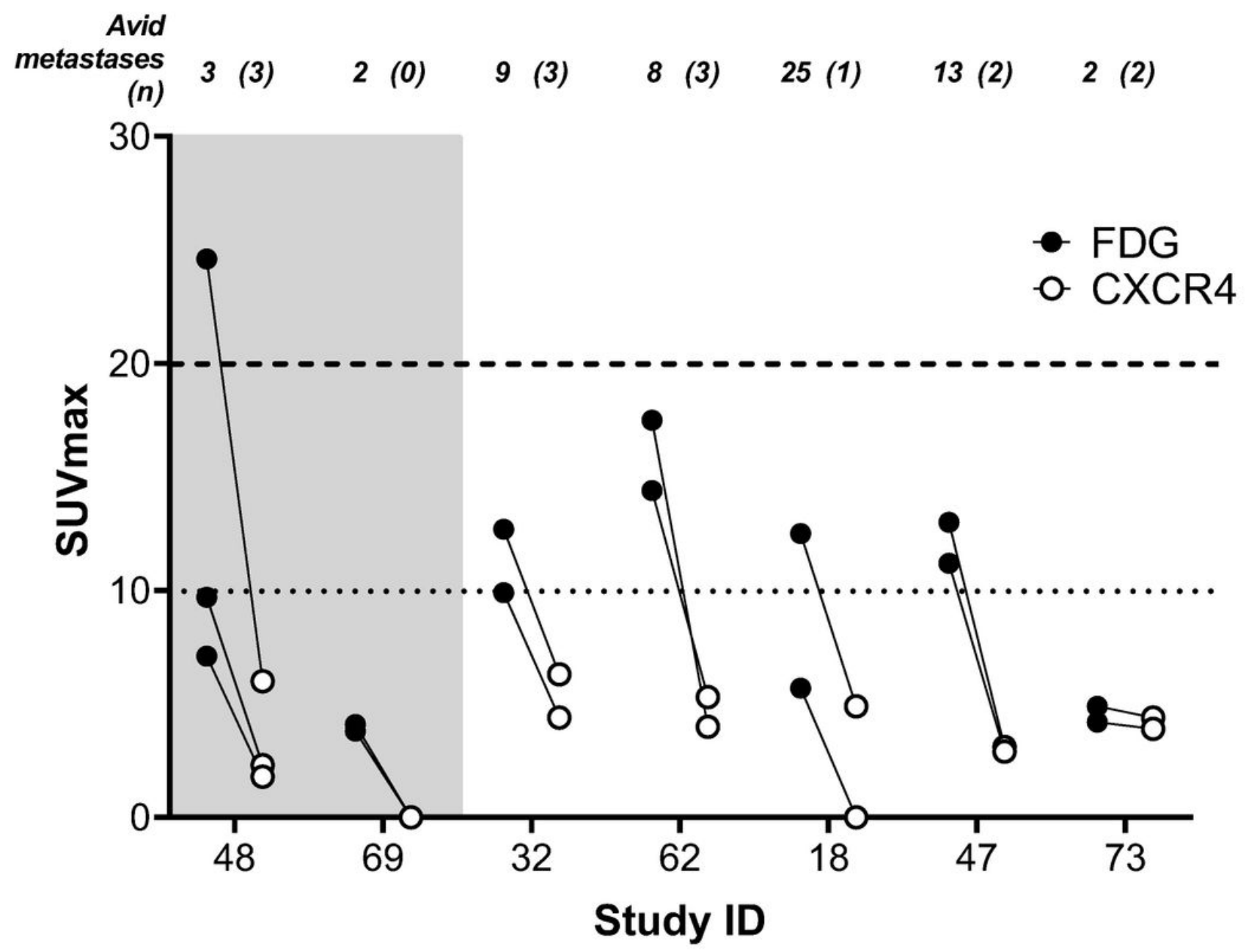

Figure 4

Maximum standardised uptake value (SUVmax) comparing FDG (where done) (closed symbol) and pentixafor tracer (open symbol) in distant metastatic sites for individual study participants (as indicated by the study identification number: Study ID) where these were detected by PET. Dotted and dashed horizontal lines indicate SUVmax of 10 and 20, respectively. Numbers in italics indicate the absolute number of avid metastases detected by FDG modality, while those in italics and parentheses indicate the absolute number of those detected by pentixafor modality.

\section{Supplementary Files}

This is a list of supplementary files associated with this preprint. Click to download.

- table1.pdf 